\title{
BOUNDEDNESS OF VECTOR-VALUED MARTINGALE TRANSFORMS ON EXTREME POINTS AND APPLICATIONS
}

\author{
TERESA MARTÍNEZ and JOSÉ L. TORREA
}

(Received 14 June 2002; revised 5 February 2003)

Communicated by A. H. Dooley

\begin{abstract}
Let $\mathbf{B}_{1}, \mathbf{B}_{2}$ be a pair of Banach spaces and $T$ be a vector valued martingale transform (with respect to general filtration) which maps $\mathbf{B}_{1}$-valued martingales into $\mathbf{B}_{2}$-valued martingales. Then, the following statements are equivalent: $T$ is bounded from $L_{\mathrm{B}_{1}}^{p}$ into $L_{\mathrm{B}_{2}}^{p}$ for some $p$ (or equivalently for every $p$ ) in the range $1<p<\infty ; T$ is bounded from $L_{\mathbf{B}_{1}}^{\infty}$ into $B M O_{\mathbf{B}_{2}} ; T$ is bounded from $B M O_{\mathbf{B}_{1}}$ into $B M O_{\mathbf{B}_{2}} ; T$ is bounded from $H_{\mathrm{B}_{1}}^{1}$ into $H_{\mathrm{B}_{2}}^{1}$. Applications to $U M D$ and martingale cotype properties are given. We also prove that the Hardy space $H_{\mathrm{B}}^{1}$ defined in the case of a general filtration has nice dense sets and nice atomic decompositions if and only if $\mathbf{B}$ has the Radon-Nikodým property.
\end{abstract}

2000 Mathematics subject classification: primary 60G42; secondary 60B 11, 46B20.

Keywords and phrases: Martingale transforms, Hardy spaces, $B M O$.

\section{Introduction and preliminaries}

It is undeniable that in the last 40 years martingale theory and harmonic analysis have been inspired and influenced by each other, and that, in particular, this has lead to a parallel development of both fields. Of course, many examples of this parallelism could be pointed out. But, as the closest to our aims, we would like to mention the shared concepts of Hardy spaces $H^{1}$ and the space of bounded mean oscillation $B M O$ (see [11] for the probabilistic part). Mainly from the works of Burkholder and Bourgain (see [7] and [4]), martingale transforms in probability and the Hilbert transform in harmonic analysis clearly play similar roles. Also in connection with the vector-valued Calderón-Zygmund operator theory in harmonic analysis, in [14]

Both authors were partially supported by DGICYT, Spain, under the grant BFM 2001-1188 and the European Comission via TMR 'Harmonic Analysis' ERB FMRXCT970159.

(C) 2004 Australian Mathematical Society 1446-7887/04 \$A2.00+0.00 
this similarity was further developed for vector-valued martingale transforms. It was shown that this theory has the added interest of providing some applications to the geometry of Banach spaces, as well as to classical operators in probability. But the theory developed in [14] deals only with $L^{p}$-bounded martingale transforms. The purpose of this paper is, on one hand, to complete that work with the study of their boundedness properties in the extreme cases $H^{1}$ and $B M O$, and on the other hand, the analysis of the structure of $H_{\mathbf{B}}^{1}$ itself for a given Banach space $\mathbf{B}$, and its relationship with the geometric properties of the space $\mathbf{B}$.

At this point, we should fix some notation. Let $(\Omega, \mathscr{F}, P)$ be a probability space carrying a stochastic basis $\left\{\mathscr{F}_{n}\right\}_{n \geq 1}$ (that is, a nondecreasing sequence of sub- $\sigma$-fields of $\mathscr{F}$ ). Given a Banach space $\mathbf{B}$, a sequence $f=\left\{f_{n}\right\}_{n \geq 1}$ of $\mathbf{B}$-valued random variables is a B-valued martingale relative to $\left\{\mathscr{F}_{n}\right\}$, if each function $f_{n}$ is $\mathscr{F}_{n}$-measurable (that is, it is an adapted sequence), integrable and $E_{n}\left(f_{n+1}\right)=E\left(f_{n+1} \mid \mathscr{F}_{n}\right)=f_{n}$, for every $n \geq 1$. We assume $f_{0}=0$ and denote by $E_{n}$ the operator defined as the conditional expectation to the sub- $\sigma$-field $\mathscr{F}_{n}$. In particular, $f_{n}=\sum_{k=1}^{n} d_{k} f$, where $d_{k} f$ are the 'increments' of the martingale $f$, that is, $d_{k} f=f_{k}-f_{k-1}$. The martingale $f$ is called $L_{\mathbf{B}}^{p}$-bounded if $\|f\|_{L_{\mathbf{B}}^{p}}=\sup _{n}\left\|f_{n}\right\|_{L_{\mathrm{B}}^{p}}$ is finite. For a detailed background on $\mathbf{B}$-valued martingales the reader is referred to [9].

The scalar-valued classical theory of Hardy spaces of martingales is nowadays well known, see $[11,17]$. Several generalizations of these spaces have been studied, for example Hardy spaces associated to certain martingale operators, as in [18] and the references therein, where also their duals are characterized. Given a Banach space B, $H_{\mathrm{B}}^{1}$ is defined as the space of martingales such that $\left\|f^{*}\right\|_{L^{1}}<\infty$ where $f^{*}$ stands for Doob's maximal operator, $f^{*}(\omega)=\sup _{n \geq 1}\left\|f_{n}(\omega)\right\|_{\mathbf{B}}$. Note that, since the sequence $\left\{\left\|f_{n}\right\|_{\mathbf{B}}\right\}$ is a positive submartingale, the so-called Doob's inequalities (see [10]) extend to the vector-valued setting, and we have

$$
\lambda P\left(f_{n}^{*}>\lambda\right) \leq C\left\|f_{n}\right\|_{L_{\mathrm{B}}^{1}} \text { and }\left\|f_{n}^{*}\right\|_{L^{p}} \leq C_{p}\left\|f_{n}\right\|_{L_{\mathrm{B}}^{p}}, \quad \text { for every } p>1 .
$$

As far as we know, in the vector-valued context, the theory of Hardy spaces has been developed only in particular cases, either for martingales of the form $f_{n}=E_{n}(f)$ for some function $f$, see [12], or for regular stochastic basis, see [5]. Our goal is to study the structure of $H_{\mathbf{B}}^{1}$ spaces without assuming any condition on the underlying stochastic basis. The main results in this part of our work relate the properties of this space to the geometric properties of the space $\mathbf{B}$ : we find that $\mathbf{B}$ enjoys the RadonNikodým property if and only if $H_{\mathbf{B}}^{1}$ has nice atomic decompositions and if and only if the set of martingales with a finite number of non-zero differences (Definition 2.4) is dense in $H_{\mathbf{B}}^{1}$ (Theorem 2.5). Related analytic results can be found in [2] and [3]. We recall that a Banach space $\mathbf{B}$ has Radon-Nikodým property if for any $\sigma$-additive, absolutely continuous set function $G: \mathscr{F} \rightarrow \mathbf{B}$ of bounded total variation with respect to $d P$, there exists a function $g \in L_{\mathbf{B}}^{1}$ such that $G(E)=\int_{E} g d P$ for all $E \in \mathscr{F}$ (see 
[9] for more details).

In [7], Burkholder studied the class of Banach spaces B for which there exists $p$, $1<p<\infty$, such that

$$
\left\|\varepsilon_{1} d_{1} f+\cdots+\varepsilon_{n} d_{n} f\right\|_{L_{\mathrm{B}}^{p}} \leq C_{p}\left\|d_{1} f+\cdots+d_{n} f\right\|_{L_{\mathrm{B}}^{p}}
$$

for all B-valued martingale difference sequences $d_{1} f, d_{2} f, \ldots$, all numbers $\varepsilon_{1}, \varepsilon_{2}, \ldots$ in $\{-1,1\}$, and all $n>1$ with a constant $C_{p}$ only depending on $p$. He called $U M D$ the class of Banach spaces which satisfy this property. The martingale $g=\left\{g_{n}\right\}$ given by $g_{n}=\sum_{k=1}^{n} \varepsilon_{k} d_{k} f$, is called the martingale transform of the martingale $f=\left\{f_{n}\right\}$.

We shall deal with vector-valued martingale transform operators, defined by sequences of operator-valued random variables $\left\{v_{n}\right\}$, instead of Burkholder's scalarvalued sequences $\left\{\varepsilon_{n}\right\}$ (for short $(T f)_{n}=\sum_{k=1}^{n} v_{k} d_{k} f$, see Definition 3.2). We proved in [14] that for a martingale transform operator $T$ as above the knowledge of the boundedness of the martingale transform operator in some fixed level, say, strong $p$ with $p>1$, is equivalent to the boundedness of the rest of the levels, and in particular to the boundedeness $\left\|(T f)^{*}\right\|_{L^{p}} \leq C_{p}\left\|f^{*}\right\|_{L^{p}}$ for every $p$ in the range $1 \leq p<\infty$. Our aim in this part of our work is to show that the philosophy behind this result (the knowledge of the boundedness at a certain level is equivalent to know the boundedness at the rest of the levels) can be extended to $B M O$ and $H^{1}$ spaces. In Theorem 3.4 it is proved that the martingale transform operators $T$ which are bounded at some level $L^{p}, 1<p<\infty$, are exactly those bounded between $B M O$ spaces, equivalently from $L^{\infty}$ into $B M O$ or, also equivalently, those whose maximal function takes functions from $L^{\infty}$ into functions of $B M O$. Also in this part of the work, no extra conditions are imposed on the stochastic basis. We handle two different notions of $B M O$ type spaces: $B M O_{p, \mathbf{B}}^{-}$and $B M O_{p, \mathbf{B}}$ are respectively the spaces of functions in $L_{\mathrm{B}}^{p}$ such that

$$
\|f\|_{B M O_{p . \mathrm{B}}^{-}}=\sup _{n \geq 1}\left\|\left(E_{n}\left\|f-f_{n-1}\right\|_{\mathbf{B}}^{p}\right)^{1 / p}\right\|_{\infty}
$$

and

$$
\|f\|_{B M O_{p, \mathbf{B}}}=\sup _{n \geq 1}\left\|\left(E_{n}\left\|f-f_{n}\right\|_{\mathbf{B}}^{p}\right)^{1 / p}\right\|_{\infty}
$$

are finite.

It is interesting to point out that as an intermediate step in the proof of that theorem, the boundedness of Doob's maximal function between $B M O$ spaces is proved in Lemma 3.5. This can be considered as the probabilistic version of the result for Hardy-Littlewood maximal operator established in [1]. We give an example of a function $f$ whose maximal is in $B M O$ but $f$ is not in $B M O$ (see Example 3.6), that, in particular, shows that the $B M O$ norms of $f$ and $f^{*}$ are not equivalent.

Finally we present some applications. A Banach space $B$ is said to be of martingale cotype $q$ if $S_{q} f=\left(\sum_{k=1}^{\infty}\left\|d_{k} f\right\|_{\mathrm{B}}^{q}\right)^{1 / q}$ satisfies $\left\|S_{q} f\right\|_{L^{q}} \leq C\|f\|_{L_{\mathrm{B}}^{q}}$ with $C$ a constant 
only depending on $q$. This property was introduced by Pisier, see [15] and [16]. In [19] it is proved that this property can be characterized in terms of some inequalities involving the Lusin area function. By identifying $S_{q}$ with the maximal of a $\ell^{q}$-valued martingale transform operator, see Subsection 4.1, we can apply the results in Section 3 and evaluate the behaviour of $S_{q}$ in the extremes $p=1$ and $p=\infty$. The ideas in that section yield a new characterization of the martingale cotype $q$. In fact, we prove that for a Banach space $B$, having martingale cotype $q$ is equivalent either to the fact that $S_{q}$ maps $L_{\mathbf{B}}^{\infty}$ into $B M O, S_{q}$ maps $B M O$ into $B M O$, or $S_{q}$ maps $H^{1}$ into $L^{1}$ boundedly. See also [13] for related results.

With a similar reasoning to the one developed for $S_{q}$, in Theorem 4.2 we prove a characterization of $U M D$ spaces as the ones in which signs martingale transforms are either $L^{\infty}-B M O$ or $B M O-B M O$ bounded. See Section 4.2 for the details.

The organization of the paper is as follows: in Section 2 the general theory of Hardy spaces is developed. The results concerning boundedness of martingale transform operators are collected in Section 3, and the applications are given in Section 4.

\section{Hardy spaces. Radon-Nikodým property}

Analogously to the scalar case, see [11] and [17], we define the following spaces of $\mathbf{B}$-valued martingales. Given $p, 1 \leq p<\infty$, and a Banach space $\mathbf{B}$, The Hardy space $H_{\mathrm{B}}^{p}$ is the space of martingales $f$ such that $\|f\|_{H_{\mathrm{B}}^{p}}=\left\|f^{*}\right\|_{L^{p}}<\infty$.

A B-valued martingale $f$ is called $L^{p}$-predictable, if there is an adapted nondecreasing sequence of functions $\left\{\lambda_{n}\right\}_{n \geq 0}$ such that $\left\|f_{n}\right\|_{\mathrm{B}} \leq \lambda_{n-1}, n \geq 1$, with $\left\|\lambda^{*}\right\|_{L^{p}}<\infty$. Such a sequence is called an admissible control for $f . \mathscr{P}_{\mathbf{B}}^{p}$ will denote the space of $L^{p}$-predictable martingales, endowed with the norm $\|f\|_{\mathscr{P}_{B}^{p}}=\inf _{\lambda}\left\|\lambda^{*}\right\|_{p}$, where $\lambda$ runs over all admissible controls for $f$. In particular, if we take $\mu_{n}=\inf _{\lambda} \lambda_{n}$, then $\|f\|_{\mathscr{P}_{B}^{p}}=\left\|\mu^{*}\right\|_{L^{p}}$ and $\mu$ is called the optimal control for $f$.

$\mathscr{A}_{\mathbf{B}}^{p}$ is the space of martingales such that $\|f\|_{\mathscr{A}_{\mathrm{B}}^{p}}=\left\|\sum_{n=1}^{\infty}\right\| d_{n} f\left\|_{\mathbf{B}}\right\|_{L^{p}}$ is finite. For every Banach space $\mathbf{B}$ and $1 \leq p<\infty$, it is clear that $\mathscr{P}_{\mathbf{B}}^{p}$ and $\mathscr{A}_{\mathbf{B}}^{p}$ are subspaces of $H_{\mathbf{B}}^{p}$; and $\|f\|_{H_{\mathbf{B}}^{p}} \leq\|f\|_{\mathscr{A}_{\mathrm{B}}^{p}},\|f\|_{H_{\mathrm{B}}^{p}} \leq\|f\|_{\mathscr{P}_{\mathbf{B}}^{p}}$. On the other hand, $H_{\mathbf{B}}^{p}=\mathscr{P}_{\mathbf{B}}^{p}+\mathscr{A}_{\mathbf{B}}^{p}$ as a consequence of the following well known result due to Davis (see [17]) in the scalar-valued case. The proof in the vector-valued setting is straightforward.

LEMMA 2.1 (Davis' decomposition). For any $p, 1 \leq p<\infty$ and $f \in H_{\mathrm{B}}^{p}$ there exist martingales $g$ and $h$, such that $g \in \mathscr{P}_{\mathbf{B}}^{p}, h \in \mathscr{A}_{\mathbf{B}}^{p}, f_{n}=g_{n}+h_{n}, n \geq 1$ and $\|g\|_{\mathscr{P}_{\mathrm{B}}^{p}} \leq(13+4 p)\|f\|_{H_{\mathrm{B}}^{p}},\|h\|_{\alpha_{\mathrm{B}}^{p}} \leq(4+4 p)\|f\|_{H_{\mathrm{B}}^{p}}$.

Let us recall that a stopping time related to a stochastic basis $\left\{\mathscr{F}_{n}\right\}$ is a function $v$ : $\Omega \rightarrow \mathbb{N} \cup\{\infty\}$ with $\{\nu=n\} \in \mathscr{F}_{n}$ for all $n \geq 1$. Given a martingale $f$, the martingale stopped at $\nu$ will be denoted by $f^{\nu}$, and is defined by $\left(f^{\nu}\right)_{n}=\sum_{k=1}^{n} \mathscr{X}_{(\nu \geq k)} d_{k} f$. 
DEFINITION 2.2. A B-valued martingale $a=\left\{a_{n}\right\}$, is said to be a $p$-atom, $1 \leq$ $p<\infty$, if there exists a stopping time $v$ such that $\mathscr{X}_{[v \geq n]} a_{n}=0$ for any $n \geq 1$, and $\left\|a^{*}\right\|_{L^{\infty}} \leq P(\{\nu \neq \infty\})^{-1 / p}$.

The following lemma is a slight generalization of the scalar-valued case, see [17].

LEMMA 2.3. Given a martingale $f=\left(f_{n}\right) \in \mathscr{P}_{\mathrm{B}}^{p}, 1 \leq p<\infty$, there exists a sequence of $p$-atoms $\left\{a^{k}\right\}_{k=-\infty}^{\infty}$ and a sequence of positive numbers $\left\{\mu_{k}\right\}_{k=-\infty}^{\infty} \in \ell^{p}$ such that

$$
f_{n}=\sum_{k=-\infty}^{\infty} \mu_{k} a_{n}^{k} \text { a.s., } \quad \sum_{k=-\infty}^{\infty} \mu_{k}^{p} \leq C\|f\|_{\mathcal{F}_{\mathrm{B}}^{p}}^{p} .
$$

In the case $p=1$, if $f_{n}=\sum_{k=-\infty}^{\infty} \mu_{k} a_{n}^{k}$ almost surely with $\left\{\mu_{k}\right\} \in \ell^{1}$ and $a^{k} 1$-atoms, then $f \in \mathscr{P}_{\mathrm{B}}^{1}$ and $f=\sum_{k=-\infty}^{\infty} \mu_{k} a^{k}$ in the $\mathscr{P}_{\mathrm{B}}^{1}$-norm and $\|f\|_{\mathscr{P}_{\mathrm{B}}^{1}} \sim \inf \sum_{k=-\infty}^{\infty}\left|\mu_{k}\right|$, where the infimum is taken over all possible decompositions of $f$.

PROOF. Let $f$ be a martingale in $\mathscr{P}_{\mathbf{B}}^{p}$ with optimal control $\left\{\lambda_{n}\right\}$. Define the non decreasing sequence of stopping times $\nu_{k}=\inf \left\{n: \lambda_{n}>2^{k}\right\}$ where inf $\emptyset=\infty$. Then,

$$
f_{n}=\sum_{k=-\infty}^{\infty}\left(\left(f^{\nu_{k+1}}\right)_{n}-\left(f^{\nu_{k}}\right)_{n}\right)=\lim _{m \rightarrow \infty}\left(\left(f^{\nu_{m}}\right)_{n}-\left(f^{\nu_{-m}}\right)_{n}\right) \text { a.s. }
$$

since, by using that $\left\{v_{m} \geq j\right\}=\left\{\lambda_{j}^{*} \leq 2^{m}\right\}$ and $\lambda^{*} \in L^{p}$, we have $\lim _{m \rightarrow \infty}\left(f^{v_{m}}\right)_{n}=f_{n}$ and $\left\|\left(f^{v_{-m}}\right)_{n}\right\|_{\mathbf{B}} \leq 2 n 2^{-m}$ almost surely. Define, for each $k$ such that $P\left(\left\{v_{k} \neq \infty\right\}\right) \neq 0$, $\mu_{k}=2^{k} 3 P\left(\left\{\nu_{k} \neq \infty\right\}\right)^{1 / p}$ and $a_{n}^{k}=\mu_{k}^{-1}\left(\left(f^{\nu_{k+1}}\right)_{n}-\left(f^{\nu_{k}}\right)_{n}\right)$; in other cases define $\mu_{k}=0, a_{n}^{k}=0$. The proof follows now the lines of the scalar case, see [17].

In order to get the reciprocal for $p=1$, let $f_{n}=\sum_{k=-\infty}^{\infty} \mu_{k} a_{n}^{k}$ with $\left\{\mu_{k}\right\} \in \ell^{1}$, $\left\{a^{k}\right\} 1$-atoms, and $\left\{\lambda_{n}^{k}\right\}$ being the optimal control for $a^{k}$. Then, $\left\{\sum_{k=-\infty}^{\infty}\left|\mu_{k}\right| \lambda_{n}^{k}\right\}_{n}$ is an admissible control for $f$. Since $\|a\|_{\mathscr{P}_{\mathbf{B}}} \leq 1$, we have that $\|f\|_{\mathscr{P}_{\mathbf{B}}^{1}} \leq \sum_{k=-\infty}^{\infty}\left|\mu_{k}\right|$ and that the convergence is also in the space $\mathscr{P}_{\mathbf{B}}^{1}$, because

$$
\left\|f_{n}-\sum_{k=-m}^{m-1} \mu_{k} a_{n}^{k}\right\|_{\mathbf{B}} \leq \sum_{|k|>m}\left|\mu_{k}\right| \lambda_{n-1}^{k} .
$$

These computations were valid for any decomposition of $f$, therefore $\|f\|_{\mathcal{P}_{\mathbf{b}}^{1}} \leq$ $\inf \sum_{k=-\infty}^{\infty}\left|\mu_{k}\right|$. On the other hand, by the first part of this lemma, $f=\sum_{k=-\infty}^{\infty} \mu_{k} a^{k}$ with $\sum_{k=-\infty}^{\infty}\left|\mu_{k}\right| \leq C\|f\|_{\mathscr{P}_{\mathrm{k}}}$. Then $\|f\|_{\mathscr{P}_{\mathrm{B}}^{1}}$ is equivalent to inf $\sum_{k=-\infty}^{\infty}\left|\mu_{k}\right|$ and the proof is finished.

Atoms are usually defined as functions and so they are in the scalar-valued case. However, it does depend on the geometry of the underlying Banach space in the 
vector-valued case, as the next result states. To this aim, define $\mathscr{P}_{a t, \mathbf{B}}^{1}$ to be the space of B-valued martingales $f$ such that $f_{n}=\sum_{k=-\infty}^{\infty} \mu_{k} a_{n}^{k}$ almost surely, where $\left\{\mu_{k}\right\} \in \ell^{1}$ and $a^{k}$ are special 1-atoms such that $a_{n}^{k}=E_{n}\left(a^{k}\right)$ for a measurable function $a^{k}$. The norm in $\mathscr{P}_{a t, \mathbf{B}}^{1}$ is given by $\|f\|_{\mathcal{P}_{a, \mathrm{~B}}^{1}}=\inf \sum_{k=-\infty}^{\infty}\left|\mu_{k}\right|$. Also, let us consider the following martingales.

DEFINITION 2.4. $f=\left\{f_{n}\right\}$ is a finite martingale if $f_{n}=E_{n}(f)$, and there exists $n_{0}$ such that $f$ is $\mathscr{F}_{n_{0}}$-measurable.

THEOREM 2.5. Given a Banach space B, the following sentences are equivalent:

(i) B has the Radon-Nikodým property.

(ii) Every 1-atom $\left\{a_{n}\right\}$ verifies $a_{n}=E_{n}(a)$ for certain function $a \in L_{\mathbf{B}}^{1}$.

(iii) $\mathscr{P}_{\mathbf{B}}^{1}=\mathscr{P}_{a t, \mathbf{B}}^{1}$.

(iv) $H_{\mathrm{B}}^{1}=\mathscr{P}_{a t, \mathbf{B}}^{1}+\mathscr{A}_{\mathbf{B}}^{1}$.

(v) Finite martingales are dense in $H_{\mathbf{B}}^{1}$.

PROOF. (ii) $\Rightarrow$ (iii) is due to Lemma 2.3 and (iii) $\Rightarrow$ (iv) is a consequence of Lemma 2.1. In order to prove (i) $\Rightarrow$ (ii), observe that if $\mathbf{B}$ has the Radon-Nikodým property, all atoms $a$ are of the form $a_{n}=E_{n}(a)\left(a \in L_{\mathrm{B}}^{1}\right)$, since $a^{*} \in L^{\infty}$, see [8]. Now, assume (iv). In order to show that $\mathbf{B}$ has the Radon-Nikodým property, we shall see that any martingale with $f^{*} \in L^{\infty}$ converges almost surely (see [8]). If $f^{*} \in L^{\infty}$, by using Lemma 2.1 and the hypothesis we can write $f=h+g$ with $h \in \mathscr{A}_{\mathrm{B}}^{1}$ and $g \in \mathscr{P}_{\mathbf{B}}^{1}=\mathscr{P}_{a t, \mathbf{B}}^{1}$. Therefore it is enough to show that $h$ and $g$ converge in $L_{\mathbf{B}}^{1}$, see [9], namely that $h_{n}$ and $g_{n}$ are Cauchy sequences in $L_{\mathbf{B}}^{1}$. The fact that $h \in \mathscr{A}_{\mathbf{B}}^{1}$ implies that $\sum_{k=1}^{\infty}\left\|d_{k} h\right\|_{L_{\mathrm{B}}^{1}}<\infty$ and, since $\left\|h_{n}-h_{m}\right\|_{L_{\mathrm{B}}^{1}} \leq \sum_{k=m+1}^{n}\left\|d_{k} h\right\|_{L_{\mathrm{B}}^{1}}$, we have that $\left\{h_{n}\right\}$ is a Cauchy sequence in $L_{\mathbf{B}}^{1}$. For the predictable part $g \in \mathscr{P}_{\mathbf{B}}^{1}=\mathscr{P}_{a t . \mathbf{B}}^{1}$, we have $g_{n}=\sum_{k=-\infty}^{\infty} \mu_{k} E_{n}\left(a^{k}\right)$ with $\left\{E_{n}\left(a^{k}\right)\right\}$ being 1-atoms and $\left\{\mu_{k}\right\} \in \ell^{1}$. By Lemma 2.3, the converge of that series is almost surely and in $\mathscr{P}_{\mathbf{B}}^{1}$-sense. Given $\varepsilon>0$, we choose $M$ such that

$$
\left\|g-\sum_{k=-M}^{M} \mu_{k} a^{k}\right\|_{\mathscr{P}^{\prime} \mid, \mathrm{B}}<\varepsilon / 3 .
$$

As $a^{k} \in L_{\mathbf{B}}^{1}$ we have $\tilde{g}_{M}=\sum_{k=-M}^{M} \mu_{k} a^{k} \in L_{\mathbf{B}}^{1}$. Thus the martingale $\left\{E_{n}\left(\tilde{g}_{M}\right)\right\}_{n=1}^{\infty}$ converges in $L_{\mathbf{B}}^{1}$, see [9]. Let $N$ be such that for $m, n>N$ we have

$$
\left\|E_{n}\left(\tilde{g}_{M}\right)-E_{m}\left(\tilde{g}_{M}\right)\right\|_{L_{\mathrm{B}}^{1}}<\varepsilon / 3 .
$$

Hence, by adding and subtracting $E_{n}\left(\tilde{g}_{M}\right)$ and $E_{m}\left(\tilde{g}_{M}\right)$, we get

$$
\left\|g_{n}-g_{m}\right\|_{L_{\mathrm{B}}^{\prime}} \leq 2\left\|g-\tilde{g}_{M}\right\|_{\mathcal{P}_{\mathrm{B}}}+\left\|E_{n}\left(\tilde{g}_{M}\right)-E_{m}\left(\tilde{g}_{M}\right)\right\|_{L_{\mathrm{B}}^{1}} \leq \varepsilon .
$$


If $\mathbf{B}$ has the Radon-Nikodým property, any $f \in H_{\mathbf{B}}^{1}$ converges in $L_{\mathbf{B}}^{1}$ since it is uniformly integrable. Convergence in $L_{\mathbf{B}}^{1}$ implies almost sure convergence (see $[8,9]$ ). Then, $f_{n}$ is an almost surely Cauchy sequence, that is, for almost every $\omega$, given $\varepsilon>0$ there exists $N_{0}=N_{0}(\omega)$ such that for all $n, m>N_{0}$,

$$
\left\|f_{n}(\omega)-f_{m}(\omega)\right\|_{\mathbf{B}}<\varepsilon .
$$

In particular, $\sup _{n \geq m}\left\|f_{n}(\omega)-f_{m}(\omega)\right\|_{\mathbf{B}}<\varepsilon$ for $n, m>N_{0}$. This implies that $\left\{\sup _{n \geq m}\left\|f_{n}-f_{m}\right\|_{\mathrm{B}}\right\}_{m}$ converges to 0 almost surely when $m \rightarrow \infty$. Moreover, $\sup _{n \geq m}\left\|f_{n}-f_{m}\right\|_{\mathbf{B}} \leq 2 f^{*} \in L_{\mathbf{B}}^{1}$. Given $f \in H_{\mathbf{B}}^{1}$ and $N \geq 1$, consider the martingale stopped at $N, f^{N}=\left(f_{1}, \ldots, f_{N}, f_{N} \ldots\right)$. Then, by Lebesgue's dominated convergence theorem:

$$
\left\|f-f^{N}\right\|_{H_{\mathbf{B}}^{1}}=\left\|\sup _{n}\right\| f_{n}-f_{n}^{N}\left\|_{\mathbf{B}}\right\|_{L_{\mathbf{B}}^{1}}=\left\|\sup _{n \geq N}\right\| f_{n}-f_{N}\left\|_{\mathbf{B}}\right\|_{L_{\mathbf{B}}^{1}} \rightarrow 0 .
$$

This shows (i) $\Rightarrow$ (v). Conversely, if finite martingales are dense in $H_{\mathrm{B}}^{1}$, in order to prove that $\mathbf{B}$ has the Radon-Nikodým property, we will see that any martingale $f$ with $f^{*} \in L^{\infty} \subset L^{1}$, converges almost surely by showing it is a Cauchy sequence in $L_{\mathbf{B}}^{1}$ (see $[8,9])$. Given $\varepsilon>0$ let $g$ be a finite martingale such that $\|f-g\|_{H_{\mathrm{H}}^{1}}<\varepsilon / 2$. Since $g$ is finite, there exists $N$ such that $g=\left(g_{1}, \ldots, g_{N}, g_{N}, \ldots\right)$. Then, with $n, m>N$, we have $g_{N}=g_{n}=g_{m}$ and

$$
\left\|f_{n}-f_{m}\right\|_{L_{\mathrm{B}}^{1}} \leq\left\|f_{n}-g_{n}\right\|_{L_{\mathrm{B}}^{1}}+\left\|f_{m}-g_{m}\right\|_{L_{\mathrm{B}}^{1}} \leq 2\|f-g\|_{H_{\mathrm{B}}^{1}} \leq \varepsilon .
$$

The former result shows that the density of finite martingales is related with the geometry of the underlying Banach space, and that in spaces enjoying the RadonNikodým property, finite martingales are a dense subclass of Hardy spaces. The following example shows that in general finite martingales are not dense in $H_{\mathbf{B}}^{1}$.

EXAMPLE 2.6. Consider $\Omega=[0,1]$ with Lebesgue's measure and for $n \geq 0$, let $r_{n}=\operatorname{sign}\left(\sin \left(\left(2^{n} \pi t\right)\right)\right.$ be the $n$-th Rademacher function. Let $\mathscr{F}_{n}=\sigma\left(r_{1}, \ldots, r_{n}\right)$ and $f$ be the $c_{0}$-valued martingale, defined by $f_{n}=\left(r_{1}, r_{2}, \ldots, r_{n}, 0,0, \ldots\right)$. For this martingale, each $f_{n}$ is a sequence $f_{n}=\left(f_{n}^{k}\right) \in c_{0}$, with $f_{n}^{k}=r_{k}$ for $k \leq n$ and $f_{n}^{k}=0$ otherwise. Moreover, $\sup _{n}\left\|f_{n}\right\|_{c_{0}}=1 \in L^{1}$.

Suppose that finite martingales are dense in $H_{c_{0}}^{1}$. Then, for any $\varepsilon>0$ there exists $g$ a finite martingale such that $\|f-g\|_{H_{c_{0}}^{1}}<\varepsilon$. This means, in particular, that $\left\|f_{n}^{k}-g_{n}^{k}\right\|_{L^{\prime}}<\varepsilon$ for any $n$ and $k$. Since $g$ is a finite martingale, there exists $N$ such that $g_{n}=g_{N}$ for all $n \geq N$, and therefore $g_{n}^{k}=g_{N}^{k}$ for all $k$ when $n \geq N$. With $n=N, k=N+1$, we have $\left\|f_{N}^{N+1}-g_{N}^{N+1}\right\|_{L^{1}}=\left\|g_{N}^{N+1}\right\|_{L^{1}}<\varepsilon$ and with $n=N+1$, $k=N+1$, we have $\left\|f_{N+1}^{N+1}-g_{N+1}^{N+1}\right\|_{L^{1}}=\left\|r_{N+1}-g_{N^{+1}}^{N+1}\right\|_{L^{1}}=\left\|r_{N+1}-g_{N}^{N+1}\right\|_{L^{1}}<\varepsilon$. Hence $1=\left\|r_{N+1}\right\|_{L^{\prime}} \leq\left\|r_{N+1}-g_{N}^{N+1}\right\|_{L^{\prime}}+\left\|g_{N}^{N+1}\right\|_{L^{\prime}}<2 \varepsilon$ for all $\varepsilon>0$. 


\section{Martingale transform operators}

Given $p, 1 \leq p<\infty$, and $\mathbf{B}$ a Banach space, analogously to the scalar case (see [11]) we define the $\mathbf{B}$-valued $B M O$ function spaces, $B M O_{p, \mathbf{B}}^{-}$and $B M O_{p, \mathbf{B}}, 1 \leq p<\infty$ as the spaces of functions $f \in L_{\mathbf{B}}^{p}$, such that, respectively

$$
\|f\|_{B M O_{p . \mathbf{B}}^{-}}=\sup _{n \geq 1}\left\|\left(E_{n}\left\|f-f_{n-1}\right\|_{\mathbf{B}}^{p}\right)^{1 / p}\right\|_{\infty}
$$

and

$$
\|f\|_{B M O_{p . \mathrm{B}}}=\sup _{n \geq 1}\left\|\left(E_{n}\left\|f-f_{n}\right\|_{\mathbf{B}}^{p}\right)^{1 / p}\right\|_{\infty}
$$

are finite, see [5]. Also, define $B D_{\infty, \mathbf{B}}$ as the space of martingales such that

$$
\|f\|_{B D_{\infty, \mathbf{B}}}=\sup _{k \geq 1}\left\|d_{k} f\right\|_{L_{\mathbf{B}}^{\infty}}
$$

is finite.

REMARK 3.1. In the scalar-valued case, the following facts are well known, see [11] and [17]. Their proofs go straightforward over the Banach-valued case.

(i) Any $f \in L^{\infty}$ belongs to $B M O_{p}, B M O_{p}^{-}, 1 \leq p<\infty$ and $B D_{\infty}$, and $\|f\|_{B M O_{p}}$, $\|f\|_{B M O_{p}^{-}},\|f\|_{B D_{\infty}}$ are smaller or equal than $2\|f\|_{L^{\infty}}$.

(ii) $B M O_{p}^{-}=B M O_{p} \cap B D_{\infty}$, with $\|f\|_{B M O_{\bar{p}}^{-}} \sim\|f\|_{B M O_{p}}+\|f\|_{B D_{\infty}}$, for every $1 \leq p<\infty$.

(iii) We have $\|f\|_{B M O_{p}}=\sup _{\nu} P(\nu \neq \infty)^{-1 / p}\left\|f-f^{\nu}\right\|_{L^{p}}$, where the supremum is taken over all $\mathscr{F}_{n}$-stopping times $v$. Also, a function $f \in L^{p}, 1 \leq p<\infty$ is in $B M O_{p}^{-}$if and only if there exists an adapted process $\left\{\theta_{n}\right\}_{n \geq 0}$ such that $\theta_{0}=0$ and $C_{\theta}=\sup _{n}\left\|E\left(\left\|f-\theta_{n-1}\right\|_{\mathbf{B}}^{p} \mid \mathscr{F}_{n}\right)^{1 / p}\right\|_{\infty}$ is finite. Then, $\|f\|_{B M O_{p}^{-}} \sim \inf _{\theta} C_{\theta}$.

(iv) The norm in all the spaces $B M O_{p}^{-}$is equivalent for any $1 \leq p<\infty$, although $B M O_{p}$ spaces are not equivalent in general, even in the regular case.

DEFInition 3.2. Let $\mathbf{B}_{1}$ and $\mathbf{B}_{2}$ be two Banach spaces, $\left\{\mathscr{F}_{n}\right\}_{n \geq 1}$ a stochastic basis in a probability space $(\Omega, \mathscr{F}, P)$, and $f=\left\{f_{n}\right\}$ a $\mathbf{B}_{1}$-valued martingale relative to $\left\{\mathscr{F}_{n}\right\}$. Let $\left\{v_{n}\right\}$ be a sequence of $\mathscr{L}\left(\mathbf{B}_{1}, \mathbf{B}_{2}\right)$-valued random variables, each $v_{n}$ being $\mathscr{F}_{n-1}$-measurable, $n \geq 2$, and $v_{1}$ being $\mathscr{F}_{1}$-measurable, with $\sup _{n \geq 1}\left\|v_{n}\right\|_{\infty} \leq 1$. Such a sequence $v=\left\{v_{n}\right\}$ will be called a multiplying sequence. The martingale given by $(T f)_{n}=\sum_{k=1}^{n} v_{k} d_{k} f$ is called the martingale transform of $f$ by the multiplying sequence $v$. $T$ will denote the martingale transform operator.

A martingale transform operator is $L^{p}$-bounded if for some constant $C$ and every martingale $f,\|T f\|_{L_{\mathrm{B}_{2}}^{p}} \leq C\|f\|_{L_{\mathrm{B}_{1}}^{p}}$. A simple example of $L^{p}$-bounded martingale transform operator, $1 \leq p \leq \infty$, for any Banach space $\mathbf{B}$ is defined by $(T f)_{n}=$ 
$\left(f^{v}\right)_{n}=\sum_{k=1}^{n} \mathscr{X}_{\{\nu \geq k]} d_{k} f$ with $\nu$ a stopping time. Observe that $\left\{\mathscr{X}_{\{\nu \geq k\}}\right\}$ is a nice multiplying sequence and it verifies

$$
\left\|\left(f^{\nu}\right)_{n}\right\|_{L_{\mathrm{B}}^{p}}^{p}=\sum_{k=1}^{n-1} \int_{\{v=k\}}\left\|E_{k}\left(f_{n}\right)\right\|_{\mathbf{B}}^{p} d P+\int_{[v \geq n\}}\left\|f_{n}\right\|_{\mathbf{B}}^{p} d P \leq\left\|f_{n}\right\|_{L_{\mathbf{B}}^{p}}^{p} .
$$

LEMMA 3.3. Let $p, 1 \leq p<\infty, T$ be an $L^{p}$-bounded martingale transform operator and a function $f \in L_{\mathbf{B}_{1}}^{p}$. Then the martingale $T f$ is of the form $(T f)_{n}=$ $E_{n}(T f)$ for some function $T f \in L_{\mathbf{B}_{2}}^{p}$.

PROOF. Given $f \in L_{\mathbf{B}_{1}}^{p}, 1 \leq p<\infty, f_{n}=E_{n}(f)$, then $f=\lim _{n \rightarrow \infty} f_{n}$ in $L_{\mathbf{B}_{1}}^{p}$ (see [9]). Given such a martingale and a pair of indexes $n>m$, we consider the martingale $g=\left\{g_{i}\right\}_{i \geq 1}$ defined as $g_{i}=f_{i}-f_{m}$ for $i \geq m+1$ and $g_{i}=0$ otherwise. Its martingale differences are $\left(0, \ldots, 0, d_{m+1} f, d_{m+2} f, \ldots\right)$ and then $(T g)_{i}=(T f)_{i}-(T f)_{m}$ if $i \geq m+1$ and it is 0 otherwise. Since $T$ is $L^{p}$-bounded, $1 \leq p<\infty$,

$$
\left\|(T f)_{n}-(T f)_{m}\right\|_{L_{\mathrm{B}_{2}}^{p}}=\left\|(T g)_{n}\right\|_{L_{\mathrm{B}_{2}}^{p}} \leq C\|g\|_{L_{\mathrm{B}_{1}}^{p}}=C \sup _{n>m}\left\|f_{n}-f_{m}\right\|_{L_{\mathrm{B}_{1}}^{p}},
$$

that is, $\left\{(T f)_{n}\right\}$ is a Cauchy sequence in $L_{\mathbf{B}_{2}}^{p}$ and converges to a function in $L_{\mathbf{B}_{2}}^{p}, T f$, verifying $(T f)_{n}=E_{n}(T f)$.

Consider, for each $k \geq 0$, the sequence of $\sigma$-algebras $\tilde{F}_{n}^{k}=\mathscr{F}_{k+n}, n \geq 1$, and the martingale transform operator $\tilde{T}_{k}$ with respect to them, defined by the multiplying sequence $\left\{\tilde{v}_{n}^{k}\right\}_{n=1}^{\infty}, \tilde{v}_{n}^{k}=v_{k+n}$. In particular, $\tilde{T}_{0}=T$. These operators verify the next result.

THEOREM 3.4. Given $\mathbf{B}_{1}, \mathbf{B}_{2}$ two Banach spaces and $T$ a martingale transform operator as in Definition 3.2 and $\tilde{T}_{k}$ as above, the following statements are equivalent when they hold for any $k \geq 0$ with constants independent of $k$ :

(i) For every $p, 1<p<\infty, \tilde{T}_{k}: B M O_{p, \mathbf{B}_{1}} \rightarrow B M O_{p, \mathbf{B}_{2}}$.

(ii) There exists $p, 1 \leq p<\infty, \tilde{T}_{k}: B M O_{p, \mathbf{B}_{1}}^{-} \rightarrow B M O_{p, \mathbf{B}_{2}}^{-}$.

(iii) There exists $p, 1 \leq p<\infty, \tilde{T}_{k}: L_{\mathbf{B}_{1}}^{\infty} \rightarrow B M O_{p, \mathbf{B}_{2}}^{-}$, where $L_{\mathbf{B}_{1}}^{\infty}$ is the space of all almost surely uniformly bounded $\mathbf{B}_{1}$-valued functions.

(iv) $\left\|\left(\tilde{T}_{k} f\right)^{*}\right\|_{B M O_{p}^{-}} \leq C_{p}\|f\|_{B M O_{p, \mathbf{B}_{1}}^{-}}$for some $p, 1 \leq p<\infty$.

(v) $\left\|\left(\tilde{T}_{k} f\right)^{*}\right\|_{B M O_{p}^{-}} \leq C_{p}\|f\|_{L_{B_{1}}^{\infty}}$ for some $p, 1 \leq p<\infty$.

(vi) $\tilde{T}_{k}$ are $L^{p}$-bounded for any $p, 1<p<\infty$.

(vii) $T$ is $L^{p}$-bounded for any $p, 1<p<\infty$.

(viii) $T$ is bounded from $H_{\mathbf{B}_{1}}^{1}$ into $H_{\mathbf{B}_{2}}^{1}$.

Proof. In [14] we proved that statements (vii) and (viii) are equivalent. The rest of the proof will be developed as follows: first we will prove (vii) $\Rightarrow$ (vi) $\Rightarrow$ (i) $\Rightarrow$ (ii). 
From (ii) we get (iii) and (iv) and from any of them we obtain (v). Last step will be proving (v) $\Rightarrow$ (viii).

Suppose $T$ is $L^{p}$-bounded. Given an $\tilde{F}_{n}^{k}$-martingale $\tilde{f}=\left(\tilde{f_{1}}, \tilde{f_{2}}, \ldots\right)$, by defining $f=\left(E_{1}\left(\tilde{f_{1}}\right), \ldots, E_{k}\left(\tilde{f_{1}}\right), \tilde{f_{1}}, \tilde{f_{2}}, \ldots\right)$ we obtain a $\tilde{F}_{n}$-martingale associated to $\tilde{f}$ with $\|f\|_{L_{\mathbf{B}_{1}}^{p}} \leq\|\tilde{f}\|_{L_{\mathbf{B}_{1}}^{p}}$. Then

$$
\begin{aligned}
\left\|\left(\tilde{T_{k}} \tilde{f}\right)_{n}\right\|_{L_{\mathbf{B}_{2}}^{p}} & =\left\|\tilde{v}_{1}^{k} \tilde{f_{1}}+\sum_{j=2}^{n} \tilde{v}_{j}^{k}\left(\tilde{f_{j}}-\tilde{f_{j-1}}\right)\right\|_{L_{\mathbf{B}_{2}}^{p}} \\
& =\left\|v_{k+1} E_{k}\left(\tilde{f_{1}}\right)+(T f)_{n}-(T f)_{k}\right\|_{L_{\mathbf{B}_{2}}^{p}} \leq C\|\tilde{f}\|_{L_{\mathbf{B}_{1}}^{p}} .
\end{aligned}
$$

This shows (vii) $\Rightarrow$ (vi) and in particular that every $\tilde{T}_{k}$ is $L^{p}$-bounded with respect to the corresponding stochastic basis, with the same constant than $T$, independent of $k$. By using this, it is enough to prove all the implications (except (v) $\Rightarrow$ (viii)) just for a martingale transform operator $T$ and check that the constants in statements (i)-(v) depend only on $p$ and the $L^{p}$-boundedness constant of $T$. Next step consists in proving that a $L^{p}$-bounded martingale transform operator $T$ maps $B M O_{p, \mathbf{B}_{1}}$ into $B M O_{p, \mathbf{B}_{2}}$ boundedly. Consider $f \in B M O_{p, \mathbf{B}_{1}}$ for some $p, 1<p<\infty$. By Lemma 3.3 the martingale $\left\{(T f)_{n}\right\}$ is of the form $(T f)_{n}=E_{n}(T f)$ where $T f$ is a function in $L_{\mathrm{B}_{2}}^{p}$. In order to see that $T f$ is in $B M O_{p, \mathbf{B}_{2}}$, we will use the characterization in Remark 3.1. Let $\nu$ be a stopping time. We have

$$
\left\|T f-(T f)^{v}\right\|_{L_{\mathbf{B}_{2}}^{p}}=\sup _{n \geq 1} \sum_{k=1}^{n-1} \int_{[v=k]}\left\|(T f)_{n}-(T f)_{k}\right\|_{\mathbf{B}_{2}}^{p} d P,
$$

and for each pair $n>k$,

$$
\int_{l v=k\}}\left\|(T f)_{n}-(T f)_{k}\right\|_{\mathbf{B}_{2}}^{p} d P=\left\|(T h)_{n}\right\|_{L_{\mathbf{B}_{2}}^{p}}^{p},
$$

where the sequence with zeros in the first $k$ coordinates $\left(0, \ldots, 0, d_{k+1} f \mathscr{X}_{(v=k)}\right.$, $\left.d_{k+2} f \mathscr{X}_{l v=k\}}, \ldots\right)$ is $h$ 's martingale differences. Since $T$ is $L^{p}$-bounded, the conditional expectation properties give

$$
\begin{aligned}
\left\|(T h)_{n}\right\|_{L_{\mathbf{B}_{2}}^{p}}^{p} & \leq C \sup _{n \geq 1}\left\|h_{n}\right\|_{L_{\mathbf{B}_{1}}^{p}}^{p}=C \sup _{n>k} \int_{\{v=k\}}\left\|E_{n}(f)-f_{k}\right\|_{\mathbf{B}_{1}}^{p} d P \\
& \leq C\|f\|_{B M O_{p, \mathbf{B}_{1}}}^{p} P(\{\nu=k\}) .
\end{aligned}
$$

and therefore

$$
\left\|T f-(T f)^{\nu}\right\|_{L_{\mathbf{k}_{2}}^{p}}^{p} \leq C\|f\|_{B M O_{p, \mathbf{B}_{1}}}^{p} P(\{\nu \neq \infty\}) .
$$

These calculations show that if a martingale transform operator is $L^{p}$-bounded relative to some stochastic basis, then it maps $B M O_{p, \mathbf{B}_{1}}$ into $B M O_{p, \mathbf{B}_{2}}$ with a constant that 
depends only on $p$ and the constant of its $L^{p}$-boundedness. Observe that martingale transform operators are always bounded in $B D_{\infty}$, since $\left\|v_{k} d_{k} f\right\|_{\mathbf{B}_{2}} \leq\left\|d_{k} f\right\|_{\mathbf{B}_{1}}$ almost surely. This, together with Remark 3.1 and (i), gives us (ii). The implications (iii) $\Rightarrow$ (ii) and (iv) $\Rightarrow$ (v) are consequences of Remark 3.1, and (iv) $\Rightarrow$ (ii) and (iii) $\Rightarrow$ (v) are due to the following lemma, whose proof will be given later.

LEMMA 3.5. If $f \in B M O_{p, \mathbf{B}}^{-}, 1 \leq p<\infty$, then $f^{*}=\sup _{n}\left\|E_{n}(f)\right\|_{\mathbf{B}} \in B M O_{p}^{-}$ and $\left\|f^{*}\right\|_{B M O_{\bar{p}}^{-}} \leq C\|f\|_{B M O_{p, \mathrm{~B}}^{-}}$.

Finally, we shall see that if $\tilde{T}_{k}$ verify (v) for any $k \geq 0$ with a constant independent of $k$, then $T$ is bounded in $H^{1}$. Observe that it is enough to prove $H^{1}$-boundedness for finite martingales, since

$$
\left\|(T f)^{*}\right\|_{L^{1}}=\sup _{n \geq 1}\left\|\left(T\left(f^{n}\right)\right)^{*}\right\|_{L^{1}} \leq \sup _{n \geq 1} C\left\|\left(f^{n}\right)^{*}\right\|_{L^{1}}=C\left\|f^{*}\right\|_{L^{1}}
$$

where $f^{n}=\left(f_{1}, f_{2}, \ldots, f_{n}, f_{n}, \ldots\right)$. Given a finite $f=\left(f_{1}, \ldots, f_{N}, f_{N}, \ldots\right)$ in $H_{\mathbf{B}_{1}}^{1}$, by Remark 2.1 there exist martingales $g \in \mathscr{P}_{\mathbf{B}_{1}}^{1}$ and $h \in \mathscr{A}_{\mathbf{B}_{1}}^{1}$ such that $f=g+h$. Since $\left\|h_{m}-h_{n}\right\|_{L_{\mathbf{B}_{1}}^{1}} \leq \sum_{k=n+1}^{m}\left\|d_{k} h\right\|_{L_{\mathbf{B}_{1}}^{1}} \rightarrow 0$, there exists a function $h \in L_{\mathbf{B}_{1}}^{1}$ such hat $h_{n}=E_{n}(h)$ for all $n \geq 1$. Then $g_{n}=f_{n}-h_{n}=E_{n}\left(f_{N}-h\right)$ for all $n \geq 1$. Moreover, $\|g\|_{\mathscr{S}_{\mathbf{B}_{1}}^{1}}+\|h\|_{\mathscr{A}_{\mathbf{B}_{1}}^{1}} \leq C\|f\|_{\boldsymbol{H}_{\mathbf{B}_{1}}^{1}}$. Now, since clearly $\left\|(T h)^{*}\right\|_{1} \leq\|h\|_{\mathscr{A}_{\mathbf{R}_{1}}^{1}}$, it is enough to prove $\left\|(T g)^{*}\right\|_{1} \leq C\|g\|_{\mathscr{P}_{\mathbf{B}_{1}}}$. By the proof of Lemma 2.3, the 1atoms of the decomposition of $g$ are defined as $a_{n}^{k}=\mu_{k}^{-1}\left(\left(g^{\nu_{k+1}}\right)_{n}-\left(g^{v_{k}}\right)_{n}\right)$ where $v_{k+1}$ and $v_{k}$ were certain stopping times. Due to the $L^{p}$-boundedness of stopped nartingales (1), that $g_{n}=E_{n}(g)$ for certain $g \in L_{\mathbf{B}_{1}}^{1}$ and Lemma 3.3, we conclude that $x_{n}^{k}=E_{n}\left(a^{k}\right)$ for certain $a^{k} \in L_{\mathbf{B}_{1}}^{\infty}$, for each $k$. Since the series converge almost surely, and each $v_{k}$ is a bounded lineal operator, we have $(T g)_{n}=\sum_{k=-\infty}^{\infty} \mu_{k}\left(T a^{k}\right)_{n}$ and $\left\|(T g)^{*}\right\|_{L^{1}} \leq \sum_{k=-\infty}^{\infty} \mu_{k}\left\|\left(T a^{k}\right)^{*}\right\|_{L^{1}}$. It will be enough to prove then that $\left\|(T a)^{*}\right\|_{L^{1}}$ are ıniformly bounded when $a$ is a 1 -atom given by a function. For $a$ a 1-atom, $(T a)^{*}=$ $(T a)^{*} \mathscr{X}_{(\nu \neq \infty)}$ for some stopping time $\nu$, and $\int_{[v=k]}(T a)^{*} d P=\int_{[\nu=k\}}\left(\tilde{T}_{k} a\right)^{*} d P$. The ast step is using (v) (recall that the boundedness constant of $\tilde{T}_{k}^{*}$ is uniform in $k$ ) and hat $\{\nu=k\} \in \mathscr{F}_{k} \subset \widetilde{F}_{1}^{k}$, to get the desired inequality

$$
\begin{aligned}
\left\|(T a)^{*}\right\|_{L^{1}} & =\sum_{k=1}^{\infty} \int_{\{v=k\}}(T a)^{*} d P=\sum_{k=1}^{\infty} \int_{\{v=k\}} E\left(\left(\tilde{T}_{k} a\right)^{*} \mid \tilde{F}_{1}^{k}\right) d P \\
& \leq \sum_{k=1}^{\infty}\left\|\left(\tilde{T}_{k} a\right)^{*}\right\|_{B M O_{1}^{-}} P(\{v=k\}) \leq C .
\end{aligned}
$$

A related version of this extreme point argument can be found in [18, Theorem 12]. 'et us now proceed with the proof of the boundedness of Doob's maximal function etween $B M O$ spaces. 
PROOF OF LEMMA 3.5. Consider the martingale transform operator $M$ given by the multiplying sequence $\left\{w_{k}\right\}_{k \geq 1}$, where $w_{k}(x)=(0, \ldots, 0, x, x, x, \ldots)$ is an element of $\ell_{\mathbf{B}}^{\infty}$ with zeros in the first $k-1$ coordinates, for any $x \in \mathbf{B}$. Then for a $\mathbf{B}$-valued martingale $f=\left\{f_{n}\right\}_{n \geq 1}$ Doob's inequality gives, for $p>1$,

$$
\left\|(M f)_{n}\right\|_{L_{\ell_{\mathrm{B}}^{\infty}}^{p}}=\left\|\left(f_{1}, \ldots, f_{n}, f_{n}, \ldots\right)\right\|_{L_{e_{\mathrm{B}}^{\infty}}^{p}}=\left\|f_{n}^{*}\right\|_{L^{p}} \leq C\left\|f_{n}\right\|_{L_{\mathrm{B}}^{p}},
$$

that is, $M$ is $L^{p}$-bounded with a constant only depending on $p$. Observe that, by using the fist part of the proof of Theorem 3.4, this implies that $M$ maps functions in $B M O_{p, \mathbf{B}}^{-}$into functions in $B M O_{p, \ell_{\mathrm{B}}^{-}}^{-}$. The proof is finished by observing that, since $f^{*}-f_{n-1}^{*} \leq \sup _{n \leq k}\left\|f_{k}-f_{n-1}\right\|_{\mathbf{B}}$, and by Remark 3.1 with $\theta_{n}=f_{n}^{*}$, we have

$$
\left\|f^{*}\right\|_{B M O_{p, \mathrm{~B}}^{-}} \leq \sup _{n \geq 1}\left\|E_{n}\left(\left(f^{*}-f_{n-1}^{*}\right)^{p}\right)\right\|_{\infty}^{1 / p} \leq\|M f\|_{B M O_{p, \ell_{\mathrm{B}}^{-\infty}}^{-}} .
$$

The converse to this lemma is not true in general, as it is shown by the following.

EXAMPLE 3.6. Consider the probability space $([-1,1], \mathscr{F}, d P)$ where $\mathscr{F}$ is the Borel $\sigma$-field and $d P=d x / 2, d x$ the Lebesgue measure, on $[-1,1]$. Take $\mathscr{F}_{n}$ to be the $\sigma$-field generated by the set $A_{n}=\left[-2^{-n}, 2^{-n}\right]$ and the Borel $\sigma$-field in $A_{n}^{c}=[-1,1] \backslash A_{n}$. They clearly define a stochastic basis. The function

$$
f(x)= \begin{cases}0 & \text { if }|x|>1 \\ |\log x| & \text { if } 0<x \leq 1 \\ \log |x| & \text { if }-1 \leq x<0\end{cases}
$$

does not belong to $B M O_{1}^{-}$, while $f^{*}=|f|$ does indeed, as an easy calculation shows.

\section{Applications}

4.1. Banach spaces of martingale cotype $q$ A Banach space $B$ is said to be of martingale cotype $q, 2 \leq q<\infty$ (in short, M-cotype $q$ ) if there exist a constant $C$ such that

$$
\sum_{n=1}^{\infty}\left\|d_{n} f\right\|_{L_{\mathbf{B}}^{q}}^{q} \leq C\|f\|_{L_{\mathbf{B}}^{q}}^{q} .
$$

Every Banach space is of M-cotype $q=\infty$. The definition is due to Pisier [15]. Non-trivial M-cotype $q<\infty$ is a geometrical property of the space, since it happens if and only if the space admits an equivalent uniformly convex norm (and therefore, in particular they are reflexive spaces), see $[15,16]$. If we consider

$$
S_{q} f=\left(\sum_{k=1}^{\infty}\left\|d_{k} f\right\|_{\mathrm{B}}^{q}\right)^{1 / q},
$$


then it is clear that a Banach B is of $M$-cotype $q$ if and only if $S_{q}$ maps $L_{\mathbf{B}}^{q}$ into $L^{q}$, for any filtration. Pisier showed that this property is equivalent to have boundedness of $S_{q}$ either from $L_{\mathbf{B}}^{p}$ into $L^{p}$ for some or for every $p, 1<p<\infty$. We will see what happens in the extreme points $p=1$ and $p=\infty$ by applying Theorem 3.4 to a particular martingale transform operator whose maximal is $S_{q}$.

Define the $\ell_{\mathbf{B}}^{q}$-valued martingale transform $Q f=\left\{(Q f)_{n}\right\}$ with multiplying sezuence $v_{k} x=x e_{k}, e_{k}$ the $k$-th element of the canonical basis of $\ell_{\mathbf{B}}^{q}$ for any $x \in \mathbf{B}$, in such a way that

$$
\text { (Qf })_{n}=\sum_{k=1}^{n} v_{k} d_{k} f=\left(d_{1} f, d_{2} f, \ldots, d_{n} f, 0, \ldots\right) \in \ell_{\mathbf{B}}^{q}
$$

for any B-valued martingale $f$. Then $(Q f)^{*}=S_{q} f$ and this gives us the key for he proof of the following characterization of the $M$-cotype property (where $B M O^{-}$ stands for any of the equivalent spaces $B M O_{p}^{-}, 1 \leq p<\infty$ ).

THEOREM 4.1. For a Banach space $\mathbf{B}$ the following statements are equivalent:

(i) B has $M$-cotype $q, 2 \leq q<\infty$.

(ii) There exists a constant $C$ such that $\left\|S_{q} f\right\|_{B M O^{-}} \leq C\|f\|_{L_{\mathrm{B}}^{\infty}}$ for any function $f \in L_{\mathbf{B}}^{\infty}$ and any stochastic basis.

(iii) There exists a constant $C$ such that $\left\|S_{q} f\right\|_{B M O^{-}} \leq C\|f\|_{B M O_{\mathrm{B}}^{-}}$for any function $f \in B M O_{\mathbf{B}}^{-}$and any stochastic basis.

(iv) There exists a constant $C$ such that $\left\|S_{q} f\right\|_{L^{1}} \leq C\|f\|_{H_{\mathrm{B}}^{1}}$ for any martingale $f \in H_{\mathbf{B}}^{1}$ and any stochastic basis.

Proof. As we observed above, if $\mathbf{B}$ has M-cotype $q$, then $Q$ satisfies (vii) in Theorem 3.4 and therefore it satisfies (iv), (v) and (viii) in that theorem, which means

$$
\left\|S_{q} f\right\|_{B M O^{-}} \leq C\|f\|_{L_{\mathrm{B}}^{\infty}}, \quad\left\|S_{q} f\right\|_{B M O^{-}} \leq C\|f\|_{B M O_{\mathrm{B}}^{-}} \quad \text { and } \quad\left\|S_{q} f\right\|_{L^{1}} \leq C\|f\|_{H_{\mathrm{B}}^{1}} \text {, }
$$

with a constant that depends only on the boundedness constant of $Q$, which is inderendent of the stochastic basis. Conversely, suppose either

$$
\left\|S_{q} f\right\|_{B M O^{-}} \leq C\|f\|_{L_{\mathrm{B}}^{\infty}}, \quad\left\|S_{q} f\right\|_{B M O^{-}} \leq C\|f\|_{B M O_{\mathrm{B}}^{-}} \quad \text { or } \quad\left\|S_{q} f\right\|_{L^{1}} \leq C\|f\|_{H_{\mathrm{B}}^{1}}
$$

or any stochastic basis. Since $\left(\tilde{Q}_{k} f\right)^{*}$ is again $S_{q} f$, then we have that statements (iv), $v$ ) or (viii) in Theorem 3.4 hold with the same constant. Therefore, we have

$$
\left\|S_{q} f\right\|_{L^{q}}^{q} \leq C\|f\|_{L_{\mathrm{B}}^{q}}^{q}
$$

vith certain universal constant $C$. 
4.2. $U M D$ Banach spaces Observe that the definition given above for $U M D$ spaces, is equivalent to require $L^{p}$-boundedness of all martingale transform operators $\left(T^{\varepsilon} f\right)_{n}=\sum_{k=1}^{n} \varepsilon_{k} d_{k} f$ whose multiplying sequences are defined by signs sequences $\left(v_{k}=\varepsilon_{k}\right)$. In this context $\tilde{T}_{k}^{\varepsilon}, k \geq 0$, is again a martingale transform operator of the same type. We have the following theorem, whose proof follows the lines of the proof of Theorem 4.1.

THEOREM 4.2. For a Banach space $\mathbf{B}$ the following statements are equivalent:

(i) B is $U M D$.

(ii) There exists a constant $C$ such that $\left\|T^{\varepsilon} f\right\|_{B M O_{\bar{B}}^{-}} \leq C\|f\|_{L_{\mathrm{B}}^{\infty}}$ for any function $f \in L_{\mathbf{B}}^{\infty}$ and any sign martingale transform $T^{\varepsilon}$.

(iii) There exists a constant $C$ such that $\left\|T^{\varepsilon} f\right\|_{B M O_{\mathrm{B}}^{-}} \leq C\|f\|_{B M O_{\mathrm{B}}^{-}}$for any function $f \in B M O_{\mathrm{B}}^{-}$and any sign martingale transform $T^{\varepsilon}$.

(iv) There exists a constant $C$ such that $\left\|\left(T^{\varepsilon} f\right)^{*}\right\|_{B M O^{-}} \leq C\|f\|_{L_{B}^{\infty}}$ for any function $f \in L_{\mathbf{B}}^{\infty}$ and any sign martingale transform $T^{\varepsilon}$.

(v) There exists a constant $C$ such that $\left\|\left(T^{\varepsilon} f\right)^{*}\right\|_{B M O^{-}} \leq C\|f\|_{B M O_{\mathrm{B}}^{-}}$for an] function $f \in B M O_{\mathrm{B}}^{-}$and any sign martingale transform $T^{\varepsilon}$.

(vi) There exists a constant $C$ such that $\left\|T^{\varepsilon} f\right\|_{H_{\mathrm{B}}^{1}} \leq C\|f\|_{H_{\mathrm{B}}^{1}}$ for any martingale $f \in H_{\mathrm{B}}^{1}$ and any sign martingale transform $T^{\varepsilon}$.

\section{References}

[1] C. Bennet, R. A. DeVore and R. Sharpley, 'Weak $-L^{\infty}$ and $B M O$ ', Ann. of Math. (2) 113 (1981) 601-611.

[2] O. Blasco, 'Hardy spaces of vector-valued functions: duality', Trans. Amer. Math. Soc. 308 (1988) 495-507.

[3] O. Blasco and J. García-Cuerva, 'Hardy spaces of Banach-space-valued distributions', Matt Nachr. 132 (1987), 57-65.

[4] J. Bourgain, 'Extension of a result of Benedeck, Calderon and Panzone', Ark. Mat. 22 (1984. 91-95.

[5] - 'Vector valued singular integrals and the $H^{1}-B M O$ duality', in: Israel seminar on gec metrical aspects of functional analysis (1983/84) XVI (Tel Aviv Univ., Tel Aviv, 1984) pp. 1-23

[6] D. L. Burkholder, 'Martingale transforms', Ann. Math. Statist. 37 (1966), 1494-1504.

[7] _ 'A geometrical characterization of Banach spaces in which martingale difference sequence are unconditional', Ann. Probab. (9) 6 (1981), 997-1011.

[8] S. D. Chatterji, 'Martingale convergence and the Radon-Nikodým theorem in Banach spaces Math. Scand. 22 (1968), 21-41.

[9] J. Diestel and J. J. Uhl, Vector measures, Math. Surveys 15 (Amer. Math. Soc., Providence, 1977

[10] J. L. Doob, Stochastic processes (Wiley, New York, 1953).

[11] A. M. Garsia, Martingale inequalities. Seminar notes on recent progress, Math. Lecture Notes Se (Benjamin, New York, 1973).

[12] C. Herz, 'Bounded mean oscillation and regulated martingales', Trans. Amer. Math. Soc. 19 (1974), 199-215. 
[13] T. Martinez, 'Uniform convexity in terms of martingale $H^{1}$ and BMO spaces', Houston $J$. Math. 27 (2001), 461-478.

[14] T. Martinez and J. L. Torrea, 'Operator-valued martingale transforms', Tohoku Math. J. 52 (2000), 449-474.

[15] G. Pisier, 'Martingales with values in uniformly convex spaces', Israel J. Math. 20 (1975), 326-350.

[16] $\_$, Probabilistic methods in the geometry of Banach spaces, Lecture Notes in Math. 1206 (Springer, Berlin, 1986).

[17] F. Weisz, Martingale Hardy spaces and its applications in Fourier Analysis, Lecture Notes in Math. 1568 (Springer, Berlin, 1994).

[18] — 'Martingale operators and Hardy spaces generated by them', Studia Math. 114 (1995), 39-70.

[19] Q. Xu, 'Littlewood-Paley theory for functions with values in uniformly convex spaces', J. Reine Angew. Math. 504 (1998), 195-226.

Departamento de Matemáticas

Universidad Autónoma de Madrid

Ciudad Universitaria de Canto Blanco

28049 Madrid

Spain

e-mail: teresa.martinez@uam.es, joseluis.torrea@uam.es 
